\title{
La Institución Escolar Dispositivo Ortopédico Para Las Lecturas Tuteladas
}

\section{Educational Institutions And Support For Mandatory Readings}

\author{
Dr. Facundo Abalo
}

\begin{abstract}
Facundo Abalo - Doctor en Comunicación - Universidad Nacional de La Plata. Magister en Artes - Universidad de Buenos Aires. Profesor adjunto e investigador - UNLP/UBA. Publicó el libro "Arte y Liminalidad", además de artículos en revistas culturales, diarios nacionales y publicaciones académicas sobre la articulación entre Arte y Comunicación. Es Director de la Editorial de la Universidad de La Plata - EDULP. Secretario Académico de la Carrera de Posgrado en Periodismo Cultural y Miembro del Comité Académico del Doctorado en Comunicación.
\end{abstract}

\section{Resumen}

Se intentará desarrollar aquí el modo en que la escuela surge como el lugar central donde se imponen las destrezas y disposiciones necesarias para la recepción de los textos escritos; la institución que va a aparecer en la vida de estos sujetos tallando sus experiencias con la lectura y la escritura. Es importante señalar aquí que en este proceso se hará referencia a una imposición de esas destrezas y disposiciones para acceder a la lectura, y no a una "negociación" entre los sujetos y la institución acerca de los modos de acceso y las significaciones sobre los textos con los que toman contacto. La escuela aparece así como la encargada de lo que se conoce como la alfabetización de estos sujetos y, al mismo tiempo, como la reguladora del impacto subjetivo de este proceso por el cual se adquirirían los capitales necesarios para su tránsito, exitoso o no, por la institución.

Palavras-chave: institución escolar, imposición de las destrezas, lecturas tuteladas

\begin{abstract}
We will try to develop here the way in which the school emerges as the central place where the skills and necessary arrangements for the reception of written texts; impose the institution that will appear in the life of these subjects carving their experiences with reading and writing. It is important to note here that in this process will be referred to an imposition of these skills and provisions
\end{abstract}


for access to reading, and not a "bargain" between the subject and the institution about access modes and meanings on texts that take contact. The school appears as responsible for what is known as the literacy of these subjects and, at the same time, as the regulator of the subjective impact of this process whereby it would acquire the necessary capital for its transit, successful or not, by the institution.

Keywords: school, imposition of skills, sheltered reading 
problematizar las condiciones de acceso, o aquello a lo que se accede.

\section{Introducion}

Una serie de investigaciones en el campo de la pedagogía que dan cuenta de cómo el aprendizaje de la escritura puede iniciarse antes de la mediación de la enseñanza formal -instancias en que algunos niños insertos en contextos sociales de producción e interpretación de la lengua escrita intuitivamente comienzan las exploración de las reglas de funcionamiento de la misma -, en la totalidad de las entrevistas realizadas en el presente trabajo los sujetos señalan a la escuela como el lugar fundacional de su contacto con la lectura y la escritura.

Dentro de estos estudios, cuyas características son más bien de corte pedagógico, se puede organizar dos grandes grupos:

1) Las investigaciones que se preocuparon por describir las habilidades de un sujeto que adquiere conocimientos (aquí podrían señalarse los aportes de la psicología y sus desarrollos sobre los aspectos sensorio-motrices).

2) Las investigaciones dedicadas a la problematización sobre el aspecto metodológico relacionado a cómo se enseña a leer y a escribir. Es decir, subyacen en los dos casos una visión técnica instrumental sobre la lectura y la escritura, sin

\section{1 - La Función Reguladora De La Escuela}

A su vez, resulta imposible referirse a la función reguladora de la escuela sin explicar la centralidad de aquello que se denominó lectoescritura en el proyecto de constitución de la Nación Argentina: el rol que desempeñaron las instituciones escolares en este proceso y el modo que se concentró en ellas - de manera concreta y efectiva- un modelo de país que depositó en la educación la tarea de modelar el ser nacional.

Desde mediados del siglo XIX y la primera mitad del siglo XX, la escuela pública en Argentina se presenta como la institución por excelencia encargada de garantizar el progreso, tarea no menor en un país que se refundaba al calor de unos acontecimientos, que lejos de desplegarse sobre territorios vírgenes en lo material y lo simbólico, necesitaron virginizarlos de manera coercitiva. La escuela, de esta manera, se convertiría en el ámbito social específicamente diseñado para reorganizar el pensamiento de unos sujetos que serían los encargados de llevar adelante el ideal civilizatorio y garantizar la reorganización del sistema social. Sujetos considerados en tanto masa homogénea a la que había que esculpir, instruir y formar, contra 
aquella otra idea que habría sostenido la barbarie como el impedimento u obstáculo de un progreso - siempre lineal- hacia la civilización.

El rol de la escuela en la constitución del Proyecto Nacional Argentino ha merecido numerosos análisis desde diferentes disciplinas que dieron cuenta de los distintos modos de funcionamiento institucional y cristalización de unos ideales forjados alrededor de la cultura escrita. Esta historia podría dividirse en varias etapas, cada una de ellas con sus particularidades territoriales, personas que encarnaron en nombre propio esos ideales y modelos pedagógicos que supusieron. Sin embargo, hubo un fenómeno que mereció especial atención por sus implicancias ideológicas, pregnancia y poder multiplicador: lo que se conoció dentro del sistema educativo argentino como el normalismo y la proliferación de las Escuelas Normales.

Más allá o más aquí del modelo pedagógico que las llamadas Escuelas Normales impulsaron, el perfil de alumno promovido y el modo en que entendieron a la cultura escrita, cabe señalarse la contundencia con que agruparon todo su arsenal de mecanismos regulatorios al servicio de la construcción de unas demarcaciones que haciendo honor a su nombre- rápidamente se erigieron como rígidas normas, en el sentido de condición de acceso y permanencia, dentro de las institución escolar. En este sentido, la construcción de las normas de funcionamiento del sistema escolar fue creando en el mismo movimiento a aquellas anormalidades que habría que identificar, señalar, encapsular, normalizar o bien expulsar en el caso de que no fuera posible su asimilación a un proyecto que tendería a borrar las diferencias, no en pro de un plan de igualdad, sino más bien de un plan de igualación. El signo de la inclusión se trazo allí con el mismo gesto de la violencia que lo nombraba, ya que lo que no decía y omitía ese ideal normativo era a través de qué mecanismos se iba construyendo esa norma y cuántos otros iban quedando fuera de ella.

De cualquier modo, se concurriría a una simplificación errónea y lineal suponer que este proceso en Argentina se llevó adelante únicamente a través de mecanismos coercitivos, a la manera de una dominación vertical. La prolífica expansión de este modelo educativo no podría obtener éxito sin el acuerdo de grandes sectores de la población que vieron en él la materialización de sus aspiraciones y deseos personales. De esta forma, la histórica relación entre el camino hacia el progreso, por un lado, y la educación como la mejor herramienta para lograr ese cometido, por el otro, se fue ramificando en un país en vías de construcción, atravesado por fuertes procesos migratorios y con disputas alrededor de la consolidación de un Proyecto Nacional:

En el litoral y en los grandes centros urbanos,
los hijos de los inmigrantes fueron los
principales "pobladores" del Normalismo.
Estos inmigrantes traían una decidida
voluntad de superación económica y social, que
si no se hacía efectiva en ellos mismos, debia 
lograrse, al menos, en la generación siguiente: la de sus hijos. (Leoz, 2004: 35)

De esta forma, el Normalismo encontró una especial interlocución entre los hijos de inmigrantes, los sectores que se desplazaban del campo a los principales centros urbanos, y específicamente en las mujeres que desempeñaron un rol central en su constitución. Todos ellos, de algún modo encarnaron esos ideales que sostuvieron a la educación como ritual de pasaje hacia unas mejores condiciones de vida, y a la escuela como la institución consagrada para llevarlos adelante. Para Gladys Leoz:

Los "bidalgos pobres de provincia", los hijos de los inmigrantes y la mujer, tuvieron un denominador común: la fe en el progreso personal por obra y gracia de la escuela. Todos creyeron ellos directamente o sus padres- que por obra del estudio, en la Escuela Normal en este caso, progresarían económica, social y culturalmente.

Habia en ellos, consciente o no, una coincidencia plena con los caracteres que dieron origen al Normalismo: fe en el progreso, fe en el uso de la razón, fe en la ilustración como motor esencial de hombres y pueblos. (2004: 35)

La fuerte política alfabetizadora de ese momento no poseía solamente como objetivo la mera enseñanza de los rudimentos básicos de la lecto-escritura, sino la configuración de nuevas

\footnotetext{
${ }^{1}$ Durante este período existían dos instrumentos legales que se ubicaron al servicio de la regulación del modelo del Normalismo: la Ley 1420 y el reglamento para la
}

Augusto Guzzo Revista Acadêmica, 2015, №15, 23-34 formas de pensamiento que posibilitarían la reorganización del sistema social de acuerdo a unos intereses específicos. La proliferación y posterior celebración de las Escuelas Normales en el territorio argentino, destinadas al mismo tiempo a la formación de maestros y maestras de educación primaria elemental (hecho que señala un fenómeno que no se cerró en sí mismo, si no que se potencia a partir de su efecto multiplicador), serviría de principal instrumento de reproducción de un modo particular de entender la lectura y la escritura al servicio del Proyecto Nacional.

Asimismo, las escuelas primarias $\mathrm{y}$ secundarias, impregnadas de un marcado positivismo europeizante y atravesado por las teorías evolucionistas, eran vividas como el paso imprescindible para el acceso a la educación universitaria, destinada a la formación de lo que se pensaba sería la clase dirigente del país. De este modo, asistimos a la ramificación de un arsenal de instituciones creadas alrededor y amparadas por ese campo que poco explicita acerca de los supuestos que encarna, conocido como el sistema educativo ${ }^{1}$.

A la par del despliegue de un proyecto político y económico de expansión territorial, se consolidaba un sistema educativo que serviría como malla de contención y corset regulador para la

Escuela Normal de Maestros de la Provincia de Buenos Aires (1877).

www.fics.edu.br 27 
formación de unos sujetos pensados como actores claves para llevar adelante el tan ansiado progreso. Como ya se ha mencionado, este proceso se expandió y consolidó gracias a la articulación de mecanismos que funcionaron como instancias de subjetivación de un orden impuesto político, económico y simbólico.

Así, y como bien señala Néstor García Canclini, emerge una dimensión central actuante entre el poder económico y el poder represivo, y que el autor denomina el Poder Cultural. Éste posee como función principal imponer a los sujetos normas culturales a través de una estrategia que combina la imposición con la disimulación, de manera tal que legitima una particular estructura dominante y la hace aparecer como natural, borrando su carácter de construcción situada; al mismo tiempo que "presenta dicha estructura como requisito y condición necesaria para la socialización de esos sujetos” (1982: 79). En este sentido, la Escuela va operando a través del Poder Cultural para la oferta de unos capitales construidos alrededor de la lectura y la escrituraque se presentan como iguales para todos e imprescindibles para un recorrido exitoso por los pliegues de la estructura que la misma institución diseña.

Se establece así una relación de manera directa entre la escolaridad y las prácticas de lectura, es decir que la "poca lectura" o la ausencia de la práctica se relaciona de manera inequívoca con la debilidad del sistema escolar o el fracaso de la escolarización. La poca lectura (o la que no está atravesada por el libro como objeto) es considerada así como una lectura no certificada o no sancionada por la institución legitimadora: la Escuela.

Cabe mencionar aquí que a muchos de los entrevistados en el marco de esta investigación les costaba reconocerse como lectores, a pesar que habían tenido contacto a lo largo de su trayectoria biográfica con distintos materiales como revistas, historietas o periódicos (ver entrevistas). El hecho que no se trate de un material que circule al interior de la escuela o no haya sido proporcionado por sus maestras con un fin didáctico, era el principal motivo por el cual consideran que "eso no era lectura", y por tanto que no los había interpelado/constituido como lectores.

Es indiscutible la centralidad que ocupó y ocupa la palabra escrita en nuestra sociedad, y cómo la Escuela, y a partir de ella, la lectura y los libros, aparecen envueltos en un aura de piadosa e incuestionable neutralidad, pensados como instrumentos puros que allanan el camino hacia el progreso. Como bien se ha expresado, la Escuela apareció durante todo período fundacional (y por supuesto, mucho más allá de él) como la garantía para democratización de los saberes a partir de la profundización, consolidación y expansión del proyecto de la alfabetización universal. Pero, como todo proyecto de universalización, la peligrosa trampa que encerró el desarrollo de esa política fue la invisibilización, la disimulación del 
modo en que se fue trazando ese universal investido de aparentes buenas intenciones de progreso; los sujetos que fueron tomados y pensados como encarnación de ese ideal universal, pensado como los modelos de hombre y mujeres que necesita la Nación, y el proceso a través del cual ese dispositivo escolar fue borrando a esos otros particulares difíciles de encuadrar dentro de la norma, pensada aquí como letrada, pero que claramente se disponía como regla o pauta de funcionamiento social.

Siguiendo a Pierre Bourdieu tomamos aquí esta idea a partir de la cual un capital cultural engendra hábitos y prácticas culturales; entonces resulta evidente cómo la lectura y la escritura aparecen como:

\begin{abstract}
una caja de resonancia de un conjunto de prácticas que se internalizan en el cuerpo de estos sujetos a la manera de esquemas de percepción y acción, es decir, el modo en cada uno de ellos entenderá el mundo y operará sobre él, en el sentido de reproducir un orden anterior, o de producir la posibilidad de un orden nuevo. (2008: 92)
\end{abstract}

Se volverá sobre este punto al momento de presentar las entrevistas, de manera tal, que resultará especialmente interesante observar las tensiones que pueden reconstruirse entre la inscripción de estos sujetos en un orden otro, es decir, su inclusión dentro del registro de la norma con la consecuente reproducción en muchos casos de las condiciones de sus propia exclusión, y esos Augusto Guzzo Revista Acadêmica, 2015, №15, 23-34 otros momentos de pliegue donde parecería aflorar un orden propio a contrapelo de los señalado desde esa misma norma.

Retomando a Bourdieu y el provocador análisis planteado en Los herederos, los estudiantes y la cultura, investigación realizada junto a Jean Claude Passeron y que se convirtiera rápidamente en un clásico para explicar la modalidad a través de la cual el sistema escolar elimina a los hijos de las clases sociales desfavorecidas, resulta de interés recuperar aquí la tesis que desarrolla cómo esta escuela -que se enuncia desde una inclusión universal y que esgrime este ideal liberador- se articula con toda una serie de prácticas y mecanismos de violencia simbólica que legitiman las relaciones de dominación de unos sujetos sobre otros, y al mismo tiempo refuerza la teoría que: "algunos bienes culturales (en este caso la lectura y la escritura) se ofrecen solo a aquellos sujetos que ya cuentan con capitales para apropiárselos antes de su ingreso a la institución” (Bourdieu y Passeron, 2009: 73).

A pesar de sus declamados enunciados de igualdad y sus principios universalizantes, este modelo de escuela pública obligatoria que, como bien se ha señalado, cumplió un rol fundamental en la construcción de ciudadanos para un modelo de Nación, vio en la diversidad de su alumnado un obstáculo para la concreción de su proyecto político-educativo. Si se partía del principio consagrado en la Ley a partir del cual todos los individuos eran iguales ante las instituciones, la www.fics.edu.br 29 
escuela retomó ese umbral, pero lejos de considerar las diferencias, puso en funcionamiento un sofisticado mecanismo de homogenización. De esta manera, aducía ofrecer las mismas herramientas para todos, pero al mismo tiempo ocultaba el dispositivo de normalización a partir del cual sostuvo la creencia que la diversidad atentaba de algún modo contra la unidad nacional. Para construir al ciudadano, era condición indispensable la eliminación de las anomalías y la reconfirmación de las normalidades funcionales a esos objetivos.

Resulta llamativo cómo ha ido cristalizándose un conjunto de representaciones que fueron asociando - de manera mecánica sin la debida problematización al respecto- el acceso a la institución escolar con la ampliación de posibilidades; y la adquisición de unos capitales que, por ser ofrecidos a todos de la misma manera, se creyó que favorecerían a resolver las asimetrías sociales. Concreciones todas sostenidas en el imaginario que ya será señalado y que define a la educación como ruta de acceso y superación de los sectores desfavorecidos.

Tanto para Bourdieu como Passeron, las instituciones escolares ponen en funcionamiento un sistema de reconocimientos y certificaciones que consagran a los sujetos ubicados en situaciones de privilegio cultural, social y económico, de manera tal que quienes circulan exitosamente por la Escuela son aquellos que ocupan previamente posiciones de exención frente a las puertas de ingreso, y que sin embargo son presentados como poseedores de "dones naturales de inteligencia". La noción de "acción escolar igualitaria" de esta manera fue dejando paso a eso que los autores señalaron como "acción escolar igualadora", en tanto tendió a nivelar tomando como piso a aquellos que ya se encontraban en situaciones favorecidas de base (2009: 36). A ello, los autores agregan que:

$$
\begin{aligned}
& \text { los estudiantes más favorecidos no deben solo a } \\
& \text { su medio de origen bábitos, entrenamientos y } \\
& \text { actitudes que les sirven directamente en sus tareas } \\
& \text { académicas, heredan también saberes y un saber } \\
& \text { hacer, gustos y un buen gusto cuya rentabilidad } \\
& \text { académica aun siendo indirecta, no por eso resulta } \\
& \text { menos evidente. (2009: } 38 \text { ). }
\end{aligned}
$$

A través de una aguda descripción, ambos autores explican los mecanismos que denominaron la elección de los elegidos para señalar los dispositivos a través de los cuales "la escuela premiaba a aquellos que ya habían sido previamente beneficiados en el reparto de capitales materiales y simbólicos en un espacio social más amplio" (2009: 45). Esto supuso la valoración de aquello pensado en términos de "sensibilidades" en las miradas más románticas, o “aptitudes" en las miradas más darwinianas, para incorporar aquello que la escuela señalaba como novedoso al enseñarlo, es decir, sostenida la escena de unos capitales que son presentados como inaugurales, pero que sin embargo ocultan 
la perversión de que ya están abí antes de ser aprendidos.

A la manera de un mago que crea la ficción de un objeto que aparece en el vacío, la escuela exhibe aparentemente para todos por igual el carácter inaugural de las herramientas para el acceso a la lectura y escritura, pero disimula que esas herramientas ya se hallan en la mochila de sus alumnos antes de su paso por el aula. De este modo, según Bourdieu, "los sistemas escolares reproducían y premiaban, bajo la adjudicación de desigual capacidad intelectual o interés frente al conocimiento lo que, en realidad, eran las consecuencias de las asimetrías sociales que coronaban simbólicamente” (2009: 35).

Según esta descripción, los sujetos únicamente actuarían en el orden de la reproducción de una estructura, dado que consciente o inconscientemente, se enfrentan a un orden que parecería inmodificable. Sin embargo, en las entrevistas realizadas para este trabajo, pueden observarse los momentos en que estos sujetos trans despliegan cierto posicionamiento singular, que logra a la manera "táctica" escamotear algo del orden establecido y realizar un tipo de apropiación particular de los materiales de lectura puestos a disposición por la institución. Asimismo, conviene aclarar que sintetizar estas instancias solamente como actos emancipatorios, sería incurrir en un error en dirección contraria, es decir, supondría concentrarse en las victorias de estos sujetos oprimidos e invisibilizar las condiciones efectivas de coerción a las que fueron sometidos en una relación signada por la asimetría: "El sufrir y experimentar la subordinación de sus porvenir profesional a una institución que a través del diploma, monopoliza un medio esencial del éxito social” (Bourdieu y Passeron, 2009: 45).

A partir del trabajo de campo desarrollado para el presente trabajo, puede observarse en las/los entrevistados/as una especie de tensión entre su discurso y el discurso institucional de la Escuela, de modo tal que se convirtió en una premisa de vital importancia pesquisar el entramado de las voces que los atraviesan y reconstruir las marcas de ese diálogo entre la voz propia y las voces otras que aparecen habitando su discurso.

La fuerte presencia de un discurso institucional, normativo, regulador $\mathrm{y}$ homogeneizante, posibilita que los momentos de producción de un orden propio se vean amenazados o debilitados, por fuerza de esa voz. otra que irrumpe para reforzar un orden de cosas dado. Esas instancias podrían definirse como momentos de ventriloquia, es decir, cuando el discurso del sujeto es habitado por otros hasta el punto de borrar cualquier tipo de posicionamiento subjetivo sobre aquello que se enuncia. Michel de Certeau hace referencia a "la alteración del lenguaje por una posesión", trazando una analogía con las personas que durante la Inquisición se pensaban como tomadas por algún espíritu externo: 
En las personas posesas existe una indeterminación del lugar desde donde bablan y que se presenta siempre como una "otra cosa" que babla en mí. Habla una especie de otro indeterminado [...]. Por un lado no se sabe quien habla o de que habla; por el otro, se tiene un saber que tiende a reclasificar la alteridad que se presenta. (1994: 237)

Asimismo, de Certeau advierte en este mismo escrito sobre los peligros de la interpretación del discurso del Otro, denunciando la violencia que se ejerce sobre la palabra del entrevistado en los intentos de transcripción al lenguaje académico.

Es importante aquí observar cómo el sujeto va relacionándose con la lengua de todos, es decir, de qué manera en muchos casos violenta su propio lugar desde lo que dice, en el momento que sostiene ese discurso otro aún a costa de su propia supervivencia ¿Con quiénes dialogan estos sujetos? ¿En qué momento entra en conflicto con aquello que constituye su propia subjetividad? ¿Cómo vive el sujeto esta experiencia? Este conjunto de interrogantes debe necesariamente articularse con una premisa central: eso que estos sujetos hacen, piensan o sienten, pero fundamentalmente aquellos que dicen, hacen piensan o sienten, tiene un sentido para ellos, y es precisamente la pregunta por el sentido de esas prácticas lo que ha guiado el interés de esta investigación. Es decir, indagar acerca del sentido que estos/estas sujetos trans le dan a la lectura.
"Mamá siempre, siempre, incentivaba el estudio y me decía: El estudio va a ser tu pasaporte a donde vos quieras llegar'. Me quedó grabado eso desde que era chiquitita. Me decía que habia que estudiar porque el que estudia triunfa, que el estudio es la clave del éxito, que el estudio hasta el día que me muera nadie me lo va a quitar. $Y$ asi fue. Con los años pienso cuánta razón tenía mi madre [...]”."

En este fragmento puede detectarse la vinculación casi directa, referenciada entre el estudio y la movilidad social. La entrevistada se halla fuertemente atravesada por el discurso letrado que encuentra en la Escuela el pasaporte para poder "ser alguien en la vida", tal como ella dice. Asimismo remarca con descredito la condición de "iletrado" de su padre:

$$
\begin{aligned}
& \text { “Mi mamá tiene estudios universitarios } \\
& \text { incompletos. Mi papá solo el primario. Yo } \\
& \text { siempre pensaba: No voy a ser como mi papá, } \\
& \text { ni voy a tener un novio que sea un iletrado'. Yo } \\
& \text { la veía a mi mamá siempre elegante y quería ser } \\
& \text { como ella. Siempre con tacos, cartera. La veíay } \\
& \text { pensaba: 'cuando sea grande voy a ser así. Ese } \\
& \text { era mi sueño, ser como ella. Y mi mamá era } \\
& \text { muy inteligente, muy inteligente”. }
\end{aligned}
$$

Posteriormente, relata la situación de ingreso durante su niñez a un colegio de hombres, a pesar de saber que ese sería un territorio hostil para el despliegue de su condición sexual. La lógica del prestigio de la institución educativa aparece entonces a contrapelo de su propio deseo:

Una entrevistada narra: 
"Yo igual quería ir a ese colegio porque abi no iba cualquiera, era super prestigioso, y el prestigio era lo que a mi me importaba. Me lo banco, me como todos mis secretos, finjo con tal de sobrevivir. Era importante para mi por la formación que me podian dar, por la herramientas que iba a incorporar con todo el tema del estudio".

Tal como afirma Bourdieu, la escuela aparece así como "el lugar que condensa todas las expectativas y promete satisfacerlas confundiendo los valores de éxito social con los de prestigio cultural" (2008: 67). Al mismo tiempo, los sujetos le asignan un lugar incuestionable al saber, al punto que parece que es el dispositivo escolar el que posee poder explicativo y nominal sobre su propio lugar en el espacio social.

Junto a Passeron explica que:

Si los propios interesados viven raramente su aprendizaje como renuncia o renegación es porque los saberes que deben conquistar son altamente valorados por la sociedad global y porque esta conquista simboliza el acceso a la elite. Así, hay que distinguir entre la facilidad para asimilar la cultura transmitida por la escuela (mucho mayor a medida que sube el origen social) y la propensión a adquirirla que alcanz̧a su máxima intensidad en la clase media. Aunque el deseo de ascenso a través de la educación no sea menos intenso en las clases inferiores que en las medias, sigue siendo irreal o abstracto dado que las posibilidades de satisfacerlo son infimas. (2009: 39)
En este sentido, otra entrevistada manifiesta que en la escuela buscaba la "explicación” moral de una normatividad que era vivida como extraña a la experiencia:

"En las horas libres siempre me ponía a leer cosas, o iba a la biblioteca. Era justo el momento cuando me habian empezado a surgir algunas dudas sobre algo interno, por ejemplo cuál era la definición que tenía una persona que se viste de otra manera o siente de otra manera. Eso me quedó de cuando era chiquita, que mi mamá me decía que cuando no entendiera algo lo buscara en el diccionario, y a mi me parecía que abi, en los libros, yo iba a encontrar lo que era [...]. Yo buscaba algo que me dijera si estaba mal o estaba bien, si tenía algún problema en la cabeza."

\section{Consideraciones Finales}

Pese a que hasta aquí se han señalado las inscripciones de estas entrevistadas dentro del discurso de la norma, accionando en algo que podríamos provisoriamente definir como rasgos del orden de la reproducción, en desarrollos posteriores podremos observar cómo estas mismas entrevistadas lograron efectuar ciertos desplazamientos que podrían identificarse dentro del "orden de la producción" de algo propio. A pesar de encontrarse en contacto con algunos materiales de lectura que la institución escolar ha suministrado como parte del canon de aquello que "debía leerse" y con una codificación normatizada sobre su interpretación, pudieron realizar una 
lectura en clave personal de ciertos textos clásicos, anclada en su experiencia subjetiva. Es decir, lo que interesa aquí es el modo en que, a pesar de estar reguladas por lo que la institución requería de ellas, en otras entrevistas se presentarán algunos datos obtenidos en el trabajo de campo que revelan esos puntos de fuga del discurso normatizado.

aclarar, como ya se ha dicho, que estos señalamiento últimos no pretenden concentrarse simplemente en las victorias de estos sujetos, sino más bien presentar la tensión existente en su propio discurso entre la reproducción de un orden que los oprime y la producción un orden singular.

\section{Bibliografía}

BOURDIEU, P y Passeron, J. C. Los herederos, los estudiantes y la cultura. Buenos Aires: Siglo XXI Editores, (2009).

DE CERTEAU, M. (1994). La escritura de la Historia. México: Universidad Iberoamericana 2006.

GARCÍA Canclini, N. Las Culturas Populares en el Capitalismo. México: Nueva Imagen, 1982.

LEOZ, G. Cien años de formalismo en el imaginario social argentino. En Hermes $\mathrm{N}^{\circ} 25$. San Luis: Instituto de Formación Docente Continua, 2004.
LITTAU, K. Teoría de la lectura. Libros, cuerpos y bibliomanía. Buenos Aires: Ediciones Manantial, 2008.

KENNARD, J. Ourself bebind ourself: A Theory for Lesbian Readers. Baltimore: The John's Hopkins University Press, 1984.

KOESTENBAUM, W. Wilde's Hard Labour and the Birth of Gay Reading. Baltimore: The John's Hopkins University Press, 1990.

KORNBLIT, A. L. (coord.). Metodologías cualitativas en ciencias sociales: modelos y procedimientos de análisis. Buenos Aires: Biblos, 2004.

SCHMUCLER, H. Un proyecto de comunicación/cultura. En Comunicación y Cultura, N¹2. México: Galerna, 1984.

VERÓN, E. La semiosis social: fragmentos de una teoría de la discursividad. Barcelona: Gedisa, 1988.

WILlIAMS, R. Teoría Cultural. En Marxismo y literatura. Barcelona: Península, 1980. 